\title{
Construcción y validación de una escala de actitudes hacia palomas domésticas en estudiantes universitarios
}

\section{Construction and validation of a scale to measure attitudes towards feral pigeons in college students}

\author{
Karina Conto Escobar ${ }^{1}$ \\ Universidad Peruana de Ciencias Aplicadas
}

Recibido: $20-08-19$

Aceptado: $17-12-19$

\section{Resumen}

Se construyó una Escala tipo Likert para medir actitudes hacia las palomas domésticas y se recopilaron evidencias de confiabilidad y validez para respaldar su uso en investigaciones psicológicas y educacionales. La validez de la Escala se evaluó mediante Juicio de Expertos y se obtuvieron valores de Coeficiente V de Aiken superiores a 0.8 para todos los ítems. La confiabilidad se evaluó mediante el método de consistencia interna, aplicando la Escala a una muestra de estudiantes de Estudios Generales de la Universidad Nacional Mayor de San Marcos, y se obtuvieron valores de Coeficiente Alfa de Cronbach superiores a 0.8 para la Escala total y para las sub escalas cognitiva, afectiva y conativa. Se concluyó que la Escala de 44 ítems es un instrumento válido y confiable para evaluar actitudes de estudiantes universitarios.

Palabras clave: Paloma doméstica; actitud; escala de Likert; validez; confiabilidad.

\begin{abstract}
A Likert Scale to measure attitudes towards feral pigeons was developed and evidence regarding reliability and validity was gathered to support its use in psychological and educational research. The validity of the Scale was evaluated by means of expert judges, and Aiken's V Coefficient values higher than 0.8 were obtained. The reliability was evaluated by means of internal consistency method, applying the Scale to a sample of students from "Universidad Nacional Mayor de San Marcos", and Cronbach's Alpha Coefficient values higher than 0.8 were obtained for the total scale and the cognitive, affective, and conative sub scales. In conclusion, the 44 item Scale is a valid an reliable instrument to evaluate university student's attitudes.
\end{abstract}

Keywords: Feral pigeon; attitude; Likert scale; validity; reliability.

1 Docente en Universidad Peruana de Ciencias Aplicadas. E-mail: karina.conto@gmail.com

(C) Los autores. Este artículo es publicado por la Revista de Investigación en Psicología de la Facultad de Psicología, Universidad Nacional Mayor de San Marcos. Este es un artículo de acceso abierto, distribuido bajo los términos de la licencia Creative Commons Atribucion - No Comercia_Compartir Igual 4.0 Internacional. (http://creativecommons.org/licenses/by-nc-sa/4.0/) que permite el uso no comercial, distribución y reproducción en cualquier medio, siempre que la obra original sea debidamente citada. 
La paloma doméstica (Columba livia var. doméstica) es un ave cosmopolita que se ha adaptado exitosamente a los ambientes urbanos, donde interacciona con las personas y contribuye a que mantengan un contacto cercano con la naturaleza para desarrollar comportamientos pro-ambientales. Sin embargo, cuando sus poblaciones son muy abundantes, se presentan afectaciones a la salud pública y ambiental que impactan negativamente en los ecosistemas urbanos, generan pérdidas económicas y disminuyen los niveles de bienestar de sus habitantes, situación que ha conllevado a que estas aves sean categorizadas como plagas urbanas (Ministerio de Salud, 2015). Algunas investigaciones han identificado que ciertas conductas ciudadanas, como alimentar directamente a las palomas domésticas o abandonar residuos alimentarios en la vía pública, contribuyen en gran medida a la proliferación de estas aves porque incrementan la cantidad de alimento disponible; y que las campañas de comunicación y educación orientadas a modificar dichas conductas constituyen una estrategia efectiva y sostenible a largo plazo que permite controlar y reducir el tamaño de sus poblaciones y sus efectos colaterales (Stock y Haag-Wackernagel, 2016; Senar, 2016). Pero para que estas campañas cumplan con su objetivo, deben incluir estrategias y contenidos apropiados para las actitudes, valores, creencias, preferencias, hábitos y/o normas sociales de la población a la cual van dirigidas (Reddy et al., 2017).

Las actitudes, en particular, son uno de los constructos psicológicos más estudiados debido a que para garantizar la persistencia de la conducta deseada a largo plazo, es necesario lograr actitudes fuertes (Casaló y Escario, 2018). Asimismo, dado que las actitudes preexistentes de una persona contribuyen a determinar la forma en que se asimila y se procesa nueva información (Briñol et al., 2007), el conocer las actitudes de la población es fundamental durante las etapas de diseño y ejecución de dichas campañas, en las que se deben adecuar los mensajes y las estrategias para minimizar la oposición y evitar conflictos al abordar temas controversiales. Además, juegan un rol importante durante la etapa de evaluación, ya que la medición de las actitudes antes y después de este tipo de iniciativas evidencia si los objetivos han sido cumplidos, y si se debe continuar con la estrategia planteada, modificarla o descartarla (Jacobson et al., 2015). Por esta razón, se considera necesario investigar las 'actitudes hacia las palomas domésticas' de la población como paso previo al diseño de estrategias de educación y/o comunicación para la modificación de conductas ciudadanas que tengan un impacto directo en la problemática socio-ambiental relacionada.

\section{Marco Teórico}

La presente investigación toma como base los conceptos planteados por Eagly \& Chaiken (1993); Zanna \& Rempel (1988) y Horcajo et al. (2015) para definir la 'actitud hacia las palomas domésticas' como: un constructo mental unitario referido a la tendencia psicológica que una persona expresa al evaluar a las 
palomas domésticas con algún grado de preferencia o antipatía; que integra información cognitiva, afectiva y/o conductual respecto a estas aves; y que puede influenciar y/o expresarse a través de las cogniciones, respuestas afectivas y/o intenciones conductuales hacia ellas. Su estructura se fundamenta en el Modelo Tridimensional o Tripartito, según el cual, el constructo unitario 'actitud' está constituido por tres dimensiones de carácter evaluativo que pueden ser definidas independientemente (Ajzen, 2005; Pallí \& Martínez, 2011; Briñol et al., 2007):

\section{Dimensión cognitiva}

Constituida por el conjunto de cogniciones que una persona posee sobre las palomas domésticas. Puede conocerse mediante el análisis de las creencias que relacionan a estas aves con características positivas o negativas, creencias sobre la forma en que influyen en el logro de objetivos personales, y creencias sobre qué tan deseable es alguna conducta asociada con ellas.

\section{Dimensión afectiva o evaluativa}

Constituida por los sentimientos y/o emociones asociados a las palomas domésticas. Puede conocerse a través de las expresiones de gusto o disgusto, aprecio o rechazo, interés o desdén que una persona evidencia en relación a estas aves.

\section{Dimensión conativa o conductual}

Constituida por el conjunto de conductas, intenciones o disposiciones a la acción relacionadas a las palomas domésticas. Puede conocerse a través de lo que las personas dicen que hacen, planean hacer o harían en situaciones que involucren a estas aves.

\section{Antecedentes}

A nivel internacional se tienen escasas investigaciones sobre actitudes hacia palomas domésticas, sus impactos sobre la salud y el ambiente, o las medidas de control de sus poblaciones; y las investigaciones existentes emplean instrumentos de medición diversos, de los cuales se desconocen sus propiedades psicométricas.

Seabra y Oliveira (2016) diseñaron y aplicaron encuestas y entrevistas a estudiantes y trabajadores de una escuela en Brasil para conocer sus actitudes hacia la presencia de palomas domésticas; conocimiento sobre problemas para la salud humana y ambiental, medidas de control y los métodos de prevención; y las conductas que contribuyen a la permanencia de estas aves en el ambiente escolar.

Harris (2016) diseñó y administró un cuestionario en línea a 226 miembros del personal administrativo y académico de la Universidad de Sudáfrica para evaluar actitudes, opiniones y percepciones sobre las palomas domésticas, sus 
actividades, si eran consideradas un problema en el campus, impactos potenciales y opciones de control.

Dos Santos (2014) diseñó y aplicó un cuestionario de 36 preguntas cerradas con formato de respuesta tipo Likert a 428 ciudadanos de Aveiro (Portugal) para conocer sus actitudes hacia las palomas domésticas, contacto personal con estas aves, problemas por excesiva concentración y posibles soluciones. El cuestionario se elaboró en base a información recopilada mediante entrevistas semi estructuradas que fue sometida a un análisis hermenéutico.

Krimowa (2012) diseñó una encuesta escrita para evaluar las actitudes hacia las palomas domésticas de una muestra aleatoria de 62 dueños y gerentes de establecimientos de comida de Wellington (Nueva Zelanda). El cuestionario constó de 16 preguntas abiertas y de opción múltiple sobre el número de palomas en la ciudad, frecuencia de avistamiento en los establecimientos, costos asociados, medidas para ahuyentarlas, y soluciones que apoyarían para el manejo de sus poblaciones.

Ryan (2011) diseñó y aplicó un cuestionario escrito de 17 preguntas abiertas y cerradas, algunas con formato de respuesta de tipo Likert, a una muestra estratificada aleatoria de 237 ciudadanos de Wellington (Nueva Zelanda) a fin de evaluar sus actitudes hacia la abundancia de palomas domésticas y sus diversos métodos de control, así como la influencia del compromiso ambiental sobre estas actitudes.

En el Perú, no se cuenta con ninguna investigación conocida sobre actitudes hacia las palomas domésticas, por este motivo, el presente trabajo propone como primer paso, la construcción de un instrumento de medición de actitudes hacia estas aves, específicamente una escala de actitudes, así como la recopilación de evidencias de validez y confiabilidad que respalden su uso para obtener información de utilidad en el diseño, ejecución y evaluación de programas de comunicación y /o educación que aborden situaciones de conflicto humano - paloma doméstica. En ese sentido, se ha decidido trabajar con estudiantes universitarios, quienes podrían constituir la primera población objetivo de dichos programas, en su calidad de futuros profesionales y referentes para otros grupos sociales que contribuyan al manejo integral de la problemática asociada a estas aves.

\section{METODO}

\section{Tipo y diseño de investigación}

El presente trabajo constituyó una investigación psicológica de tipo instrumental o tecnológica, pues buscó contribuir con un nuevo instrumento de medición de actitudes y un análisis preliminar de sus propiedades psicométricas (Ato et al., 2013). Su diseño metodológico fue no experimental transversal, ya que ocurrió sin manipulación deliberada de la variable en estudio, y la recolección y el análisis de los datos se realizaron en un momento determinado (Hernández et al., 2014). 


\section{Procedimiento}

Para la construcción y validación de la "Escala de Actitudes hacia Palomas Domésticas" se consideraron las etapas propuestas por Hernández et al. (2014) y Morales (2011).

\section{Definición de la variable}

Se identificó la variable en estudio (actitudes hacia las palomas domésticas) y se estableció su definición conceptual y operacional, la cual incluyó la identificación de sus dimensiones e indicadores según las bases teóricas del constructo 'actitud'.

\section{Revisión de la literatura}

Se llevó a cabo un análisis exhaustivo de la literatura científica sobre actitudes hacia las palomas domésticas en diferentes bases de datos (Google Académico, Scielo, Scopus, ERIC, PubMed, etc.), a fin de identificar los instrumentos de medición de actitudes más empleados y evaluarlos para determinar la conveniencia de su uso en la presente investigación.

\section{Construcción de la versión preliminar de la Escala de Actitudes}

Los ítems de la Escala fueron formulados en función a las dimensiones cognitiva, afectiva y conativa de la variable 'actitud hacia las palomas domésticas', y abarcaron temas relacionados a la biología y ecología de estas aves, impactos y posibles medidas de control de su población. Se decidió trabajar con el número tradicional de cinco categorías de respuesta (escala de 5 puntos) para expresar el grado de acuerdo con el contenido de los ítems (totalmente de acuerdo, de acuerdo, ni de acuerdo ni en desacuerdo, en desacuerdo, totalmente en desacuerdo). A cada categoría de respuesta por ítem se le asignó un valor numérico del 1 al 5 , correspondiendo 1 a la categoría que calificaba al ítem con actitud menos favorable y 5, al más favorable. Finalmente, los ítems fueron revisados por tres expertos, quienes realizaron sugerencias para mejorar la definición de la variable, sus dimensiones e indicadores, obteniéndose de esta manera la versión preliminar de la Escala.

\section{Prueba Piloto de la Escala de Actitudes}

La versión preliminar de la Escala se administró en Febrero del 2019 en los salones de clases durante los horarios de mañana y tarde ( 8 am a $5 \mathrm{pm}$ ), previa autorización de la Escuela de Estudios Generales (EEG) y la firma por escrito de un documento de Consentimiento Informado, que incluyó el objetivo de la investigación, identificación de la investigadora, la importancia de la participación de los estudiantes, posibles riesgos y cláusula de confidencialidad del manejo de datos. 


\section{Población}

La población elegida estuvo conformada por 1930 alumnos de Estudios Generales de la Universidad Nacional Mayor de San Marcos (UNMSM) matriculados en el Ciclo Verano 2019-0 (10.9\% del Área Académico Profesional de Ciencias Básicas, 4.2\% de Ciencias de la Salud, 20.6\% de Ciencias Económicas y de la Gestión, $20.7 \%$ de Humanidades y Ciencias Jurídicas y Sociales y 43.6\% de Ingenierías), $34.3 \%$ mujeres y $65.7 \%$ hombres.

\section{Muestra}

El tamaño de la muestra se calculó considerando un mínimo de 5 sujetos por cada ítem de la Escala, según lo sugerido por Nunnally (1978) y Argibay (2006), y se seleccionó a los participantes mediante un muestreo no probabilístico incidental. La muestra estuvo conformada por 256 participantes (Apéndice. Tabla 5), mayormente hombres, con una edad promedio de 19.7 años (DE: 1.7, Moda: 20), pertenecientes a 54 Escuelas Profesionales de las cinco Áreas, principalmente de la EP de Física (5.9\%), y residentes en 40 distritos del Departamento de Lima, en su mayoría en San Juan de Lurigancho (10.5\%).

\section{Análisis de ítems}

Se ingresaron las repuestas de los participantes a una matriz de datos del programa IBM SPSS ${ }^{\circledR}$ para calcular el Coeficiente de Correlación Ítem-Test corregido para los 47 ítems de la versión preliminar de la Escala, a fin de decidir qué ítems mantener y cuáles eliminar.

\section{Cálculo de la validez}

La validez de contenido se determinó mediante el cálculo del Coeficiente $\mathrm{V}$ de Aiken a partir de las valoraciones cuantitativas obtenidas mediante Juicio de Expertos. Los expertos seleccionados para tal fin fueron diez profesionales con especialidades en psicología educativa, educación superior, biología, medicina veterinaria, salud pública e impacto ambiental, quienes evaluaron los ítems en una escala dicotómica. Los criterios a evaluar fueron: a) claridad, que el ítem esté formulado en un lenguaje apropiado, y b) pertinencia, que el ítem permita medir la dimensión de la variable a la que corresponda. Luego de la evaluación de los expertos, se calculó el Coeficiente V de Aiken para cada ítem de la Escala con un nivel de significación estadística del 0.05 . Asimismo, los expertos realizaron una evaluación cualitativa de los ítems que permitió mejorar el formato general y las instrucciones, así como el orden y la redacción de los ítems, obteniéndose la versión final de la Escala de Actitudes (Apéndice. Escala de Actitudes Hacia Palomas Domésticas). 


\section{Administración de la versión final de la Escala de Actitudes}

La versión final de la Escala se administró en Mayo del 2019 en los salones de clases en los horarios de mañana y tarde ( 8 am a 5 pm) y, al igual que para la administración de la Escala preliminar, se contó con la autorización de la EEG y la firma por escrito del Consentimiento Informado. La población elegida estuvo conformada por 6479 alumnos de Estudios Generales de la UNMSM matriculados en el Ciclo 2019-1 (6.7\% de Ciencias Básicas, $16.8 \%$ de Salud, $21.1 \%$ de Económicas, $25.8 \%$ de Humanidades y $29.7 \%$ de Ingenierías), $39.6 \%$ mujeres y $60.4 \%$ hombres. El tamaño de la muestra se calculó en el programa Decision Analyst STATS ${ }^{\mathrm{TM}}$ 2.0, considerando un porcentaje de error aceptable máximo de $5 \%$, nivel de porcentaje estimado de $50 \%$ y nivel de confianza deseado de $95 \%$; y se seleccionó a los participantes mediante un muestreo no probabilístico incidental. La muestra estuvo conformada por 363 participantes (Apéndice. Tabla 5), hombres y mujeres en proporciones similares, con una edad promedio de 18.9 años (DE: 1.8, Moda: 19), pertenecientes a 63 EPs de las cinco Áreas, principalmente de las EPs de Contabilidad y Derecho (6.1\%), y residentes en 42 distritos del Departamento de Lima, en su mayoría en San Juan de Lurigancho (10.5\%).

\section{Cálculo de la confiabilidad}

Los valores obtenidos fueron ingresados a una matriz de datos del programa IBM SPSS $®$, en el que se calculó el coeficiente Alfa de Cronbach para estimar la confiabilidad de la Escala de Actitudes en general y para cada dimensión, así como el coeficiente de Correlación Ítem-Test corregido.

\section{Baremación}

La misma matriz de datos fue empleada para elaborar un baremo en puntajes percentilares de la Escala de Actitudes en el programa IBM SPSS ${ }^{\circledR}$, tanto a nivel general como para cada dimensión.

\section{RESULTADOS}

\section{Análisis de ítems}

Del total de 47 ítems de la Escala preliminar, 42 ítems presentaron valores de Coeficiente de Correlación Ítem-Test corregido entre 0.38 y 0.79 , y fueron mantenidos como parte de la Escala; mientras que 5 ítems presentaron valores inferiores entre 0.19 y 0.29 , de los cuales 3 (3, 19 y 25) fueron eliminados definitivamente, y los 2 restantes (41 y 45) fueron modificados, obteniéndose finalmente una Escala de 44 ítems válidos (Tabla 1). 
Tabla 1

Estadísticos de la versión preliminar de la Escala de Actitudes

\begin{tabular}{lcccccc}
\hline $\begin{array}{l}\text { Ítems en las } \\
\text { versiones sucesivas }\end{array}$ & $\begin{array}{c}\mathbf{N}^{\circ} \mathbf{d e} \\
\text { ítems }\end{array}$ & $\begin{array}{c}\text { Alfa de } \\
\text { Cronbach }\end{array}$ & $\begin{array}{c}\text { Alfa de Cronbach basada en } \\
\text { elementos estandarizados }\end{array}$ & Media & Varianza & $\begin{array}{c}\text { Desviación } \\
\text { estándar }\end{array}$ \\
\hline Todos los ítems & 47 & 0.961 & 0.961 & 145.48 & 736.13 & 27.13 \\
Ítem No 3 eliminado & 46 & 0.962 & 0.961 & 143.60 & 723.65 & 26.90 \\
Ítem N ${ }^{\circ}$ 19 eliminado & 45 & 0.962 & 0.962 & 140.90 & 709.59 & 26.64 \\
Ítem N ${ }^{\circ}$ 25 eliminado & 44 & 0.963 & 0.962 & 137.14 & 694.92 & 26.36 \\
\hline
\end{tabular}

\section{Validez}

Los valores del Coeficiente V de Aiken de cada uno de los ítems de la Escala final para el criterio de 'claridad' tuvieron un valor mínimo de 0.8 y máximo de 1; mientras que para el criterio de 'pertinencia', tuvieron valores entre 0.9 y 1 , en ambos casos con una significancia estadística de 0.05 (Tabla 2).

\section{Tabla 2}

Coeficiente $V$ de Aiken para los criterios de Claridad y Pertinencia de los items de la versión final de la Escala de Actitudes agrupados según dimensión.

\begin{tabular}{cccccccccccccccccccc}
\hline \multicolumn{11}{c}{ Claridad } & \multicolumn{1}{c}{ Cognitiva } & \multicolumn{1}{c}{ Afectiva } & \multicolumn{1}{c}{ Conativa } & \multicolumn{1}{c}{ Cognitiva } & \multicolumn{1}{c}{ Afectiva } & \multicolumn{3}{c}{ Conativa } \\
\hline $\mathbf{N}^{\circ}$ & $\mathbf{V}$ & $\mathbf{p}$ & $\mathbf{N}^{\circ}$ & $\mathbf{V}$ & $\mathbf{p}$ & $\mathbf{N}^{\circ}$ & $\mathbf{V}$ & $\mathbf{p}$ & $\mathbf{N}^{\circ}$ & $\mathbf{V}$ & $\mathbf{p}$ & $\mathbf{N}^{\circ}$ & $\mathbf{V}$ & $\mathbf{p}$ & $\mathbf{N}^{\circ}$ & $\mathbf{V}$ & $\mathbf{p}$ \\
\hline $\mathbf{1}$ & 1 & 0.001 & $\mathbf{3}$ & 1 & 0.001 & $\mathbf{2}$ & 1 & 0.001 & $\mathbf{1}$ & 1 & 0.001 & $\mathbf{3}$ & 1 & 0.001 & $\mathbf{2}$ & 1 & 0.001 \\
$\mathbf{4}$ & 1 & 0.001 & $\mathbf{6}$ & 1 & 0.001 & $\mathbf{5}$ & 1 & 0.001 & $\mathbf{4}$ & 1 & 0.001 & $\mathbf{6}$ & 1 & 0.001 & $\mathbf{5}$ & 1 & 0.001 \\
$\mathbf{7}$ & 1 & 0.001 & $\mathbf{8}$ & 1 & 0.001 & $\mathbf{1 3}$ & 1 & 0.001 & $\mathbf{7}$ & 1 & 0.001 & $\mathbf{8}$ & 1 & 0.001 & $\mathbf{1 3}$ & 1 & 0.001 \\
$\mathbf{9}$ & 1 & 0.001 & $\mathbf{1 0}$ & 1 & 0.001 & $\mathbf{1 5}$ & 0.8 & 0.049 & $\mathbf{9}$ & 1 & 0.001 & $\mathbf{1 0}$ & 1 & 0.001 & $\mathbf{1 5}$ & 0.9 & 0.001 \\
$\mathbf{1 1}$ & 1 & 0.001 & $\mathbf{1 2}$ & 1 & 0.001 & $\mathbf{1 7}$ & 1 & 0.001 & $\mathbf{1 1}$ & 1 & 0.001 & $\mathbf{1 2}$ & 1 & 0.001 & $\mathbf{1 7}$ & 1 & 0.001 \\
$\mathbf{1 4}$ & 1 & 0.001 & $\mathbf{1 6}$ & 1 & 0.001 & $\mathbf{1 8}$ & 1 & 0.001 & $\mathbf{1 4}$ & 1 & 0.001 & $\mathbf{1 6}$ & 1 & 0.001 & $\mathbf{1 8}$ & 1 & 0.001 \\
$\mathbf{2 0}$ & 1 & 0.001 & $\mathbf{1 9}$ & 1 & 0.001 & $\mathbf{2 1}$ & 1 & 0.001 & $\mathbf{2 0}$ & 1 & 0.001 & $\mathbf{1 9}$ & 1 & 0.001 & $\mathbf{2 1}$ & 1 & 0.001 \\
$\mathbf{2 4}$ & 1 & 0.001 & $\mathbf{2 2}$ & 1 & 0.001 & $\mathbf{2 3}$ & 1 & 0.001 & $\mathbf{2 4}$ & 1 & 0.001 & $\mathbf{2 2}$ & 1 & 0.001 & $\mathbf{2 3}$ & 1 & 0.001 \\
$\mathbf{2 6}$ & 1 & 0.001 & $\mathbf{2 5}$ & 1 & 0.001 & $\mathbf{2 7}$ & 1 & 0.001 & $\mathbf{2 6}$ & 1 & 0.001 & $\mathbf{2 5}$ & 1 & 0.001 & $\mathbf{2 7}$ & 1 & 0.001 \\
$\mathbf{3 0}$ & 1 & 0.001 & $\mathbf{2 8}$ & 1 & 0.001 & $\mathbf{2 9}$ & 1 & 0.001 & $\mathbf{3 0}$ & 1 & 0.001 & $\mathbf{2 8}$ & 1 & 0.001 & $\mathbf{2 9}$ & 1 & 0.001 \\
$\mathbf{3 4}$ & 1 & 0.001 & $\mathbf{3 1}$ & 1 & 0.001 & $\mathbf{3 2}$ & 1 & 0.001 & $\mathbf{3 4}$ & 1 & 0.001 & $\mathbf{3 1}$ & 1 & 0.001 & $\mathbf{3 2}$ & 1 & 0.001 \\
$\mathbf{3 6}$ & 1 & 0.001 & $\mathbf{3 3}$ & 1 & 0.001 & $\mathbf{3 5}$ & 1 & 0.001 & $\mathbf{3 6}$ & 1 & 0.001 & $\mathbf{3 3}$ & 1 & 0.001 & $\mathbf{3 5}$ & 1 & 0.001 \\
$\mathbf{3 8}$ & 1 & 0.001 & $\mathbf{3 9}$ & 0.9 & 0.001 & $\mathbf{3 7}$ & 1 & 0.001 & $\mathbf{3 8}$ & 1 & 0.001 & $\mathbf{3 9}$ & 1 & 0.001 & $\mathbf{3 7}$ & 1 & 0.001 \\
$\mathbf{4 1}$ & 1 & 0.001 & $\mathbf{4 0}$ & 1 & 0.001 & & & & 41 & 1 & 0.001 & $\mathbf{4 0}$ & 1 & 0.001 & & & \\
$\mathbf{4 3}$ & 0.9 & 0.001 & $\mathbf{4 2}$ & 0.8 & 0.049 & & & & $\mathbf{4 3}$ & 1 & 0.001 & $\mathbf{4 2}$ & 1 & 0.001 & & \\
& & & $\mathbf{4 4}$ & 1 & 0.001 & & & & & & & $\mathbf{4 4}$ & 1 & 0.001 & & & \\
\hline
\end{tabular}

\section{Confiabilidad}

Los valores del Coeficiente Alfa de Cronbach se encontraron por encima de 0.8 , tanto para la totalidad de los ítems de la Escala final, como para los ítems agrupados según las dimensiones de la variable en estudio (Tabla 3). 
Tabla 3

Estadísticos de la versión final de la Escala de Actitudes.

\begin{tabular}{lcccccc}
\hline Escala & $\begin{array}{c}\mathbf{N}^{\circ} \mathbf{d e} \\
\text { ítems }\end{array}$ & $\begin{array}{c}\text { Alfa de } \\
\text { Cronbach }\end{array}$ & $\begin{array}{c}\text { Alfa de Cronbach basada en } \\
\text { elementos estandarizados }\end{array}$ & Media & Varianza & $\begin{array}{c}\text { Desviación } \\
\text { estándar }\end{array}$ \\
\hline Total & 44 & 0.966 & 0.966 & 138.55 & 749.18 & 27.37 \\
Cognitiva & 15 & 0.902 & 0.902 & 45.53 & 80.17 & 8.95 \\
Afectiva & 16 & 0.925 & 0.925 & 49.94 & 118.95 & 10.91 \\
Conativa & 13 & 0.887 & 0.889 & 43.08 & 73.63 & 8.58 \\
\hline
\end{tabular}

Los valores del Coeficiente de Correlación Ítem-Test corregido para los ítems de la Escala final presentaron un valor mínimo de 0.40 y un valor máximo de 0.79 (Tabla 4).

Tabla 4

Estadísticos item-test de la versión final de la Escala de Actitudes.

\begin{tabular}{cccccccc}
\hline $\mathbf{N}^{\circ}$ & $\begin{array}{c}\text { Correlación } \\
\text { ítem-test corregida }\end{array}$ & $\mathbf{N}^{\circ}$ & $\begin{array}{c}\text { Correlación } \\
\text { ítem-test corregida }\end{array}$ & $\mathbf{N}^{\circ}$ & $\begin{array}{c}\text { Correlación } \\
\text { item-test corregida }\end{array}$ & $\mathbf{N}^{\circ}$ & $\begin{array}{c}\text { Correlación } \\
\text { item-test corregida }\end{array}$ \\
\hline $\mathbf{1}$ & 0.54 & $\mathbf{1 2}$ & 0.66 & $\mathbf{2 3}$ & 0.79 & $\mathbf{3 4}$ & 0.62 \\
$\mathbf{2}$ & 0.64 & $\mathbf{1 3}$ & 0.68 & $\mathbf{2 4}$ & 0.40 & $\mathbf{3 5}$ & 0.59 \\
$\mathbf{3}$ & 0.46 & $\mathbf{1 4}$ & 0.65 & $\mathbf{2 5}$ & 0.69 & $\mathbf{3 6}$ & 0.45 \\
$\mathbf{4}$ & 0.57 & $\mathbf{1 5}$ & 0.61 & $\mathbf{2 6}$ & 0.66 & $\mathbf{3 7}$ & 0.68 \\
$\mathbf{5}$ & 0.63 & $\mathbf{1 6}$ & 0.68 & $\mathbf{2 7}$ & 0.73 & $\mathbf{3 8}$ & 0.67 \\
$\mathbf{6}$ & 0.47 & $\mathbf{1 7}$ & 0.71 & $\mathbf{2 8}$ & 0.64 & $\mathbf{3 9}$ & 0.77 \\
$\mathbf{7}$ & 0.71 & $\mathbf{1 8}$ & 0.51 & $\mathbf{2 9}$ & 0.52 & $\mathbf{4 0}$ & 0.70 \\
$\mathbf{8}$ & 0.67 & $\mathbf{1 9}$ & 0.47 & $\mathbf{3 0}$ & 0.77 & $\mathbf{4 1}$ & 0.57 \\
$\mathbf{9}$ & 0.58 & $\mathbf{2 0}$ & 0.56 & $\mathbf{3 1}$ & 0.63 & $\mathbf{4 2}$ & 0.52 \\
$\mathbf{1 0}$ & 0.63 & $\mathbf{2 1}$ & 0.54 & $\mathbf{3 2}$ & 0.61 & $\mathbf{4 3}$ & 0.78 \\
$\mathbf{1 1}$ & 0.58 & $\mathbf{2 2}$ & 0.61 & $\mathbf{3 3}$ & 0.58 & $\mathbf{4 4}$ & 0.64 \\
\hline
\end{tabular}

\section{Baremación}

Se obtuvo el baremo percentilar de la Escala total y de sus dimensiones (cognitiva, afectiva y conativa). En la Tabla 6 se especifican los percentiles (PC), los puntajes directos (PD), la media (M) y la desviación estándar (DE).

Tabla 6

Baremo de la Escala de Actitudes Hacia Palomas Domésticas, a nivel general y según dimensiones.

\begin{tabular}{ccccc}
\hline PC & \multicolumn{5}{c}{ PD } \\
\cline { 2 - 5 } & Total & Cognitiva & Afectiva & Conativa \\
\cline { 2 - 5 } $\mathbf{7 5}$ & 155 & 51 & 56 & 48 \\
$\mathbf{5 0}$ & 139 & 45 & 51 & 43 \\
$\mathbf{2 5}$ & 121 & 39 & 43 & 37 \\
\hline $\mathbf{M}$ & 138.55 & 45.53 & 49.94 & 43.08 \\
$\mathbf{D E}$ & 27.37 & 8.95 & 10.91 & 8.58 \\
\hline
\end{tabular}


Según esta tabla, un estudiante con un puntaje total de 139 en la Escala, se ubicaría en el percentil 50 y su nivel de actitud hacia las palomas domésticas se encontraría por encima del 50\% de los estudiantes. Asimismo, un estudiante con un puntaje de 56 en la dimensión afectiva de la Escala, se ubicaría en el percentil 75 y su nivel de actitud afectiva hacia las palomas domésticas se encontraría por encima del $75 \%$ de los estudiantes.

\section{DISCUSIÓN}

La presente investigación se propuso construir una escala para medir actitudes hacia palomas domésticas, y obtener evidencias de validez y confiabilidad que sustenten su uso para generar información sobre lo que piensan, sienten y hacen las personas en relación a las palomas domésticas y la problemática socio-ambiental asociada. Esta Escala de Actitudes, y la información que puede ser obtenida a partir de ella, es de utilidad no solo para psicólogos y educadores a fin de diseñar programas de comunicación y educación, sino también para diversos profesionales que deseen desarrollar políticas públicas, intervenciones sanitarias o programas de manejo de la población de palomas, con miras a lograr la mejora de la salud de los ecosistemas urbanos, y la calidad de vida de los ciudadanos y la población urbana de palomas domésticas y otras especies de fauna y flora.

Como parte del proceso de construcción y validación de la Escala, se llevó a cabo un análisis de los ítems de la versión preliminar mediante el método de Correlación Ítem-Total. Este método permitió evaluar la relación de cada ítem con el total de ítems a través de los Coeficientes de Correlación Ítem-Test corregido. Aquellos ítems con valores elevados eran los que presentaban mayor correlación con el total de ítems y medían lo mismo que los demás (Morales, 2011). En ese sentido, se usó como referencia lo propuesto por Falvey et al. (1994), quien considera a los ítems con valores superiores a 0.3 como buenos y a los ítems con valores inferiores como inaceptables, de tal forma que aquellos ítems con valores buenos fueron mantenidos como parte de la Escala final, mientras que aquellos con valores inaceptables fueron eliminados o modificados.

Para determinar la validez de contenido de la Escala final y comprobar que este instrumento mide la variable que se había propuesto medir, se evaluó el Coeficiente V de Aiken. Los resultados obtenidos demostraron que los 44 ítems de la Escala pueden ser considerados válidos para un nivel de significación estadística de $\mathrm{p}<0.05$, puesto que presentaron valores superiores a 0.8 , tal como lo propuesto por Escurra (1988) e Hyrkäs et al. (2003). Esto significa que al menos 8 de los 10 expertos mostraron su acuerdo en relación a la pertinencia y claridad de redacción de los ítems, y evidencia que los ítems constituyen una muestra representativa de las dimensiones cognitiva, afectiva y conativa de la variable en estudio. 
Para evaluar la confiabilidad de la Escala final y determinar si produce resultados consistentes y coherentes, se calculó el Coeficiente Alfa de Cronbach de la Escala en su conjunto y de las sub escalas cognitiva, afectiva y conativa, cada una compuesta por los ítems correspondientes a las dimensiones de la variable en estudio. Los valores obtenidos en todos los casos fueron superiores a lo propuesto por algunos autores, como Garson (2013), quien establece que un valor mínimo de 0.60 es aceptable para propósitos exploratorios; Nunnally (1987), con un valor mínimo de 0.80; Morales (2007), de 0.70; o Lauriola (2003, citado en Hernández et al., 2014), de 0.90. Además, estos valores fueron similares o superiores al valor de confiabilidad promedio de 0.83 de artículos en revistas de Psicología de la Educación (Osborne, 2003). De igual manera, los valores de los Coeficientes de Correlación Ítem-Test corregido para los 44 ítems de la Escala final fueron superiores a 0.40, valores aceptables según Falvey et al. (1994), confirmando que los ítems mejorados de la versión preliminar de la Escala superaron la prueba de validación para ser incluidos en la Escala final.

En términos generales, las evidencias obtenidas mediante la presente investigación han demostrado que la Escala de Actitudes hacia Palomas Domésticas presenta niveles de validez y confiabilidad aceptables que justifican su uso. Sin embargo, es importante aclarar que los resultados aquí mostrados son más representativos de los estudiantes de Estudios Generales de la UNMSM, por lo que se recomienda que futuras investigaciones puedan emplear muestras de mayor tamaño, provenientes de diferentes centros de enseñanza, y en diferentes centros urbanos a nivel nacional, examinando diferencias de acuerdo al sexo, estrato socioeconómico, lugar de residencia, etc. Además, se recomienda recopilar evidencias de validez mediante otros métodos, como el análisis factorial.

\section{CONCLUSIONES}

1. La Escala de Actitudes hacia Palomas Domésticas está constituida por 44 ítems y mide las dimensiones cognitiva, afectiva y conativa.

2. La Escala presenta un nivel adecuado de confiabilidad por el método de consistencia interna.

3. La Escala posee un nivel adecuado de validez de contenido mediante Juicio de Expertos.

4. La Escala puede ser empleada para evaluar actitudes hacia palomas domésticas en estudiantes universitarios.

\section{NOTA DE RECONOCIMIENTO}

Se agradece a los investigadores J. Villafana, K. Melgar, J. Landázuri, B. Mendoza, R. Fernández, R. McGuire, L. Sánchez, S. Berrocal, J. Pequeña, T. Salas, R. 
Watanabe, L. Huamán, J. Yovera, R. Gutiérrez, B. Tuesta y M. Velásquez por sus valiosos aportes durante el proceso de construcción y validación de la Escala de Actitudes, así como a la UNMSM por las facilidades brindadas para la aplicación de la misma.

\section{REFERENCIAS}

Aiken, L. R. (1985). Three coefficients for analyzing the reliability and validity of ratings. Educational and Psychological Measurement, 45, 131-142. doi: $10.1177 / 0013164485451012$

Ajzen, I. (2005). Attitudes, personality, and behavior. Berkshire, UK: McGraw-Hill Education.

Argibay, J.C. (2006). Técnicas psicométricas. Cuestiones de validez y confiabilidad. Subjetividad y Procesos Cognitivos, 8, 15-33.

Ato, M., López, J. J., y Benavente, A. (2013). Un sistema de clasificación de los diseños de investigación en psicología. Anales de Psicología, 29(3), 1038-1059. doi: 10.6018/ analesps.29.3.178511

Briñol, P., Falces, C., y Becerra, A. (2007). Actitudes. En J. Morales, C. Huici, M. Moya, y E. Gaviria (Eds.). Psicologia Social (457-490). Madrid: McGraw-Hill.

Casaló, L. V., y Escario, J. J. (2018). Heterogeneity in the association between environmental attitudes and pro-environmental behavior: A multilevel regression approach. Journal of Cleaner Production, 175, 155-163. Doi: 10.1016/j.jclepro.2017.11.237

Dos Santos, A. (2014). Os Pombos e as Pessoas em Ambiente Urbano (Aveiro) (Tesis de Maestría). Universidade de Aveiro, Portugal.

Eagly, A. H., y Chaiken, S. (1993). The psychology of attitudes. US: Harcourt Brace Jovanovich College Publishers.

Escurra, L. M. (1988). Cuantificación de la validez de contenido por criterio de jueces. Revista de psicología, 6(1-2), 103-111.

Falvey, P., Holbrook J. y David C. (1994). Assessing students. Hong Kong: Longman.

Garson, G. D. (2013). Validity and reliability. Asheboro, NC: Statistical Associates Publishers.

Harris, E. (2016). Strategic interdisciplinary approach for non-lethal pigeon control on the University of South Africa's Mukleneuk campus (Tesis de Doctorado). Recuperado de: http://uir.unisa.ac.za/handle/10500/22180

Hernández, R., Fernández, C., y Baptista, P. (2014). Metodología de la investigación. México: Editorial Mc Graw Hill.

Horcajo, J., Briñol, P., Díaz, D., y Becerra, A. (2015) Actitudes: concepto, estructura y medición. En Sabucedo J. M., y Morales J. F. (Eds.). Psicología Social (117-136). Madrid: Editorial Médica Panamericana. 
Hyrkäs, K., Appelqvist-Schmidlechner, K., y Oksa, L. (2003). Validating an instrument for clinical supervision using an expert panel. International Journal of nursing studies, 40(6), 619 -625. Doi: 10.1016/S0020-7489(03)00036-1

Jacobson, S. K., McDuff, M. D., y Monroe, M. C. (2015). Conservation education and outreach techniques. UK: Oxford University Press.

Krimowa, S. (2012). Pigeons and people: resource ecology and human dimensions of urban wildlife (Tesis de Maestría). Recuperado de: http://researcharchive.vuw.ac.nz/ handle/10063/2329

Ministerio de Salud. (2015). Documento Técnico: Manual para la vigilancia, prevención y control sanitario de agentes zoonóticos y zoonosis relacionados a la paloma doméstica. Lima: Dirección General de Salud Ambiental.

Morales, P. (2011). Guía para construir cuestionarios y escalas de actitudes. Recuperado de: http://www.upcomillas.es/personal/peter/ otrosdocumentos/ Guiaparaconstruirescalasdeactitudes.pdf

Morales, P. (2007). La fiabilidad de los tests y escalas. Recuperado de: https://web. upcomillas.es/personal/peter/estadisticabasica/Fiabilidad.pdf

Nunnally, J. C. (1987). Teoría psicométrica. México: Editorial Trillas.

Osborne, J. W. (2003). Effect sizes and the disattenuation of correlation and regression coefficients: lessons from educational psychology. Practical Assessment, Research \& Evaluation, 8(11), 1-5.

Pallí, C. y Martínez, L. (2011). Naturaleza y Organización de las Actitudes. En T. Ibáñez (Ed.). Introducción a la psicología social (183-256). Aragón, Barcelona: Editorial UOC.

Reddy, S. M., Montambault, J., Masuda, Y. J., Keenan, E., Butler, W., Fisher, J. R., y Gneezy, A. (2017). Advancing conservation by understanding and influencing human behavior. Conservation Letters, 10(2), 248-256. Doi: 10.1111/conl.12252

Ryan, A. C. (2011). The distribution, density and movements of feral pigeons Columba livia and their relationship with people (Tesis de Maestría). Recuperado de: https:// researcharchive.vuw.ac.nz/xmlui/handle/10063/2045

Seabra, E. y Oliveira, E. (2016). Salud humana y efectos ambientales derivados de la presencia de palomas en el entorno urbano. Revista Cientifica Multidisciplinaria Base de Conocimiento, 2(1). 106-128.

Senar, J. C., Montalvo, T., Pascual, J., y Peracho, V. (2016). Reducing the availability of food to control feral pigeons: changes in population size and composition. Pest management science, 73(2), 313-317. Doi: 10.1002/ps.4272

Stock, B., y Haag-Wackernagel, D. (2016). Food shortage affects reproduction of Feral Pigeons Columba livia at rearing of nestlings. Ibis, 158(4), 776-783. Doi: 10.1111/ ibi.12385

Zanna, M. P., y Rempel, J. K. (1988). Attitudes: A new look at an old concept. En D. BarTal, A. Kruglanski (Eds.). The social psychology of knowledge (315-334). Cambridge, UK: Cambridge University Press. 


\section{APÉNDICE}

Tabla 5

Estadísticas descriptivas de las variables demográficas de la muestra piloto y la muestra final.

\begin{tabular}{|c|c|c|c|c|}
\hline \multirow{2}{*}{ Variables } & \multicolumn{2}{|c|}{ Piloto } & \multicolumn{2}{|c|}{ Final } \\
\hline & $\mathbf{N}$ & $\%$ & $\mathbf{N}$ & $\%$ \\
\hline \multicolumn{5}{|l|}{ Sexo } \\
\hline Hombre & 154 & 60.2 & 182 & 50.1 \\
\hline Mujer & 102 & 39.8 & 181 & 49.9 \\
\hline \multicolumn{5}{|l|}{ Área Académico Profesional } \\
\hline Ciencias Básicas & 65 & 25.4 & 24 & 6.6 \\
\hline Ciencias de la Salud & 29 & 11.3 & 61 & 16.8 \\
\hline Ciencias Económicas y de la Gestión & 51 & 19.9 & 76 & 20.9 \\
\hline Humanidades y Ciencias Jurídicas y Sociales & 41 & 16.0 & 94 & 25.9 \\
\hline Ingenierías & 70 & 27.3 & 108 & 29.8 \\
\hline \multicolumn{5}{|l|}{ Provincia } \\
\hline \multicolumn{5}{|l|}{ Lima } \\
\hline Lima Norte & 87 & 34 & 117 & 32 \\
\hline Lima Centro & 44 & 17 & 61 & 17 \\
\hline Lima Sur & 36 & 14 & 72 & 20 \\
\hline Lima Este & 61 & 24 & 83 & 23 \\
\hline Callao & 23 & 9 & 25 & 7 \\
\hline Lima Provincias & 5 & 2 & 5 & 1 \\
\hline Total & 256 & 100.0 & 363 & 100.0 \\
\hline
\end{tabular}




\section{ESCALA DE ACTITUDES HACIA PALOMAS DOMÉSTICAS}

\section{INSTRUCCIONES}

A continuación se presentan una serie de ítems o enunciados sobre palomas domésticas, ante los cuales usted debe expresar si se encuentra totalmente de acuerdo, de acuerdo, ni de acuerdo ni en desacuerdo, en desacuerdo o totalmente en desacuerdo.

Para cada ítem, marque un aspa ("X") dentro del casillero que mejor refleje su posición.

Debe marcar solo un (1) casillero por cada ítem.

Debe responder a todos los ítems.

No hay respuestas correctas o incorrectas. Responda con sinceridad y con cierta rapidez.

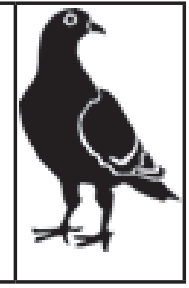

¿Qué tan de acuerdo se encuentra con los siguientes ítems?

\begin{tabular}{|c|c|c|c|c|c|c|}
\hline $\mathbf{N}^{\circ}$ & ÍTEMS & $\begin{array}{l}\text { Totalmente } \\
\text { de acuerdo }\end{array}$ & $\begin{array}{c}\text { De } \\
\text { acuerdo }\end{array}$ & $\begin{array}{c}\text { Ni de acuerdo } \\
\text { ni en } \\
\text { desacuerdo }\end{array}$ & $\begin{array}{c}\text { En } \\
\text { desacuerdo }\end{array}$ & $\begin{array}{c}\text { Totalmente } \\
\text { en } \\
\text { desacuerdo }\end{array}$ \\
\hline 1 & $\begin{array}{l}\text { La presencia de palomas domésticas mejora } \\
\text { el paisaje de la ciudad. }\end{array}$ & & & & & \\
\hline 2 & $\begin{array}{l}\text { Evitaría transitar por lugares donde hay gran } \\
\text { cantidad de palomas domésticas. }\end{array}$ & & & & & \\
\hline 3 & $\begin{array}{l}\text { Me molesta que las palomas domésticas } \\
\text { hagan nido en mi vivienda. }\end{array}$ & & & & & \\
\hline 4 & $\begin{array}{l}\text { Se debe disminuir la cantidad de palomas } \\
\text { domésticas de la ciudad. }\end{array}$ & & & & & \\
\hline 5 & $\begin{array}{l}\text { Si tuviera que tocar una paloma doméstica, } \\
\text { me negaría a hacerlo. }\end{array}$ & & & & & \\
\hline 6 & Me gustan las palomas domésticas. & & & & & \\
\hline 7 & $\begin{array}{l}\text { Los sonidos que emiten las palomas } \\
\text { domésticas perturban a las personas. }\end{array}$ & & & & & \\
\hline 8 & $\begin{array}{l}\text { Me molesta que las palomas domésticas } \\
\text { defequen en calles y edificaciones. }\end{array}$ & & & & & \\
\hline 9 & $\begin{array}{l}\text { Las palomas domésticas son perjudiciales } \\
\text { para los animales que viven en la ciudad. }\end{array}$ & & & & & \\
\hline 10 & Me gusta observar a las palomas domésticas. & & & & & \\
\hline 11 & $\begin{array}{l}\text { Debe prohibirse alimentar a las palomas } \\
\text { domésticas en la vía pública. }\end{array}$ & & & & & \\
\hline 12 & $\begin{array}{l}\text { Prefiero mantenerme alejado/a de las palomas } \\
\text { domésticas. }\end{array}$ & & & & & \\
\hline 13 & $\begin{array}{l}\text { Si encontrara una paloma doméstica herida, } \\
\text { haría algo para ayudarla. }\end{array}$ & & & & & \\
\hline 14 & Las palomas domésticas son inteligentes. & & & & & \\
\hline 15 & $\begin{array}{l}\text { Estoy dispuesto/a a deshacerme de las } \\
\text { palomas domésticas que me causen molestias. }\end{array}$ & & & & & \\
\hline 16 & $\begin{array}{l}\text { Me alegraría si la cantidad de palomas } \\
\text { domésticas en la ciudad disminuyera. }\end{array}$ & & & & & \\
\hline 17 & $\begin{array}{l}\text { Permitiría que las palomas domésticas } \\
\text { hicieran sus nidos en mi vivienda. }\end{array}$ & & & & & \\
\hline 18 & $\begin{array}{l}\text { Estoy dispuesto/a a denunciar algún caso de } \\
\text { maltrato contra palomas domésticas. }\end{array}$ & & & & & \\
\hline 19 & Me gusta alimentar a las palomas domésticas. & & & & & \\
\hline 20 & Las palomas domésticas son aves hermosas. & & & & & \\
\hline
\end{tabular}




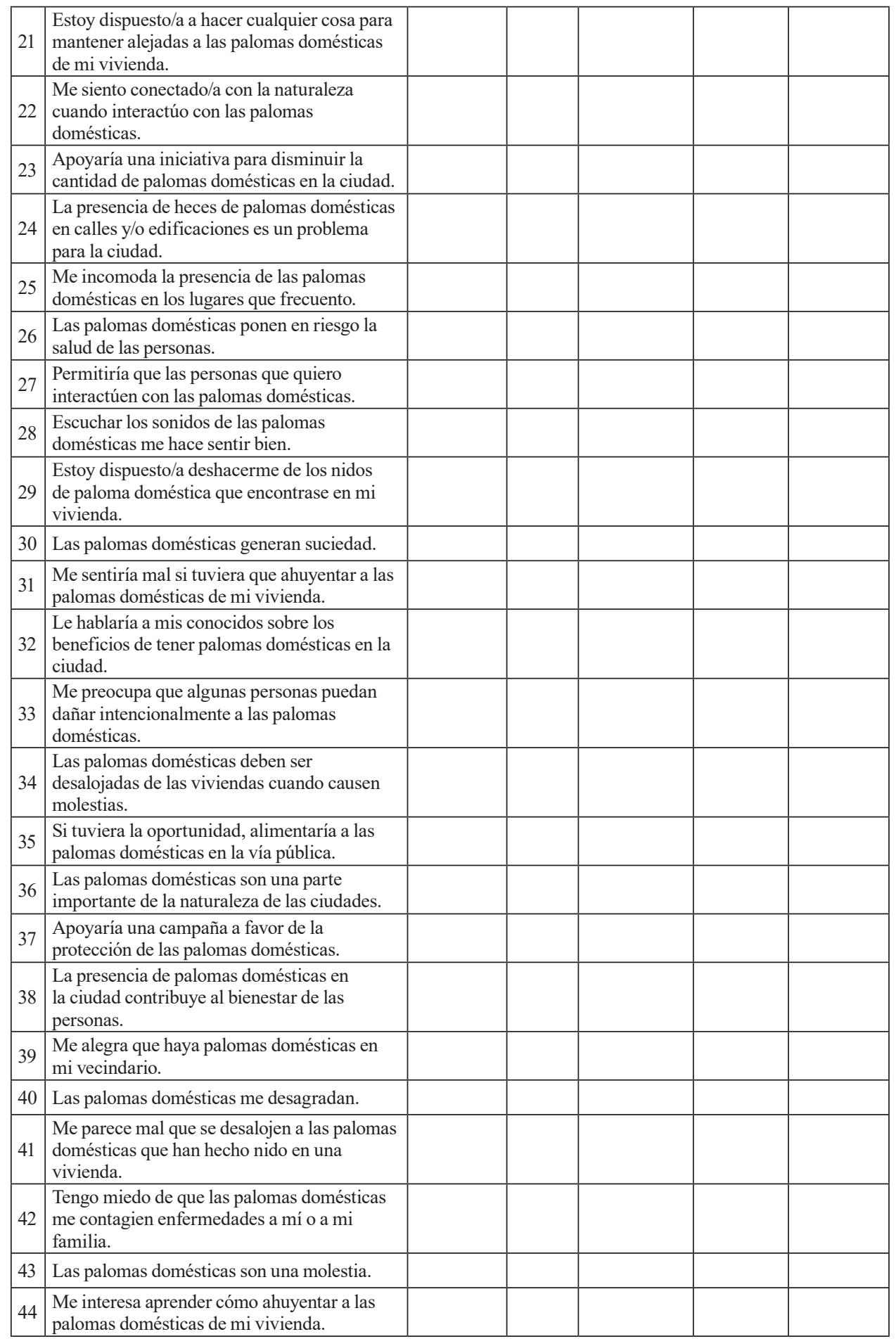

